\title{
Asociación entre caries dental de madres y de sus hijos menores de 72 meses en el centro de crecimiento y desarrollo-lactancia materna Hospital Nacional Cayetano Heredia, Lima, Perú
}

Association between dental caries in mothers and their children under $\mathbf{7 2}$ months old from the Center of Growth and Development- Breastfeeding Hospital Nacional Cayetano Heredia, Lima, Peru.

\author{
Milagros Falcon Aguilar ${ }^{1, a}$
}

\section{RESUMEN}

Objetivo: Analizar la asociación entre caries dental de madres y de sus hijos menores de 72 meses del Centro de Crecimiento y desarrollo- Lactancia materna del Hospital Nacional Cayetano Heredia en la ciudad de Lima, Perú. Materiales y métodos: Este estudio fue analítico, observacional, y transversal. Y se realizó sobre una muestra de 102 pares madre-hijo, los niños fueron menores de 72 meses de edad. Se realizaron exámenes bucales a ambos y se buscó asociación entre prevalencia de caries dental y experiencia de caries dental con otras variables como edad, sexo y grado de instrucción. Se empleó la prueba estadística de Chi-cuadrado corregida por Yates y Fisher. El estudio contó con un nivel de confianza de $95 \%$ y un $p<0,05$. Resultados: La prevalencia de caries dental en madres fue de $59.8 \%$ y en los niños de 9,8\%. En cuanto a la experiencia de caries dental, las madres tuvieron un CPOD promedio de 6,8 y un CPOS de 16,7. Los niños tuvieron un ceod promedio de 0,3 , y el mismo valor para el ceos. Conclusión: No existió asociación entre caries dental de la madre y sus hijos menores de 72 meses del Centro de Desarrollo y Crecimiento - Lactancia materna en el Hospital Nacional Cayetano Heredia, Lima- Perú.

PALABRAS CLAVE: Caries dental, experiencia de caries, prevalencia de caries. 


\section{SUMMARY}

Objective: Analyze the association between dental caries in mothers y and their children under 72 months old from the Center of Growth and Development- Breastfeeding in the Hospital Nacional Cayetano Heredia in Lima, Peru. Materials and methods: This study was analytical, observational and cross- sectional. The analysis was made with a sample of 204 subjects, 102 pairs of mothers-children, the children were under 72 months old. Oral examinations were performed to the mothers and their children, we tried to associate the prevalence of dental caries and dental caries experience with other variables such as age, sex and level of education. Chi- Square corrected by Yates and Fisher was used as a statistical test. The study had a 95\% of confidence level and $\mathrm{p}<0.05$. Results: The prevalence of dental caries in mothers was $59.8 \%$ and in their children $9.8 \%$. The mothers had an average of 6.8 DMFT and DMFS of 16.7 in dental caries. The children had an average of 0.3 ceod with the same value for the ceos. Conclusions: There was no association between dental caries in mothers and their children under 72 months of the Growth and Development Center - Breastfeeding in the Hospital Nacional Cayetano Heredia, Lima- Peru.

KEY WORDS: Dental caries, dental caries experience, dental caries prevalence.

\section{INTRODUCCIÓN}

Las enfermedades bucodentales son unas de las principales patologías en la población. Afectan la salud general, disminuyen la calidad de vida, la autoestima y pueden ocasionar dolor y ansiedad. Estas enfermedades pueden también ser el origen de enfermedades a distancia. Su tratamiento representa para la sociedad un importante consumo de medicamentos, tiempo y recursos económicos $(1,2)$

La caries dental es una enfermedad multifactorial e infectocontagiosa que está presente en aproximadamente 60 a $90 \%$ de la población escolar asiática y latinoamericana, así como en la mayoría de adultos $(3,4)$. Al tener un origen multifactorial, su prevalencia se ha visto asociada con características sociales, culturales y económicas. Existe una asociacion entre la madre y la salud bucal de sus hijos, ésta tiene influencia tanto en la forma en la que el niño va a tener responsabilidad en el cuidado de su salud oral, en la disposición para ir al dentista, y ser receptivo a la hora de recibir tratamientos odontológicos, como en la experiencia de caries $(1,2)$.

En este estudio se evaluó la caries dental a través del Índice de ceod/CPOD, el cual se calculó sumando por separado el número de superficies y el número de piezas dentales con caries dental, perdidas por caries dental y obturadas presentes en boca al momento del examen; a continuación, se sacó el promedio de ambos al dividir ambos resultados entre el número total de superficies en boca y el número total de piezas dentales presentes en boca al momento del examen, respectivamente.

El objetivo del presente estudio fue encontrar asociación entre la salud bucal de la madre y de su hijo menor de 72 meses de edad en el Centro de Crecimiento y Desarrollo - Lactancia Materna, del Hospital Nacional Cayetano Heredia, Lima, Perú, en el año 2014.

\section{MATERIAL Y MÉTODO}

La investigación fue analítica, observacional y transversal. La población de estudio fueron madres y sus niños menores de 72 meses de edad que acudieron al Centro de Crecimiento y Desarrollo- Lactancia Materna del Hospital Nacional Cayetano Heredia (HNCH), Lima- Perú en el año 2014. Según datos del Análisis situacional de salud (ASIS) del Hospital Nacional Cayetano Heredia $(\mathrm{HNCH})$ el número total de madres con hijos de la edad indicada que acudieron durante los primeros seis meses del año 2014 fue aproximadamente de 318 sujetos.

El tamaño muestralse calculómediante el programa estadístico EPIDAT 4.0 a partir del dato brindado por el Centro de Crecimiento y Desarrollo- Lactancia Materna del Hospital Nacional Cayetano Heredia del HNCH. Se determinó que el mínimo tamaño muestral para estimar la media poblacional, con una 
prevalencia del $62,3 \%$, un nivel de significancia del $95 \%$ y un $\mathrm{p}<0,05$, es de mínimo 170 sujetos. Siendo para el presente estudio una muestra de 170 niños con caries y sus respectivas madres biológicas. Sin embargo, en el presente estudio se utilizó una muestra de 102 pares madre- hijo debido a la baja afluencia de niños menores de 72 meses que acudieron al Centro de Crecimiento y Desarrollo- Lactancia Materna del $\mathrm{HNCH}$ durante los meses de noviembre y diciembre del 2014. Los sujetos de evaluación se seleccionaron al cumplir con ser niños de ambos sexos menores de 72 meses sin presencia de alguna condición sistémica que comprometiera su salud. La muestra constó de 102 niños menores de 72 meses de edad, 57 de sexo masculino y 45 de sexo femenino, todos con sus respectivas madres biológicas.

Las gestiones necesarias se realizaron a través del mismo Hospital Nacional Cayetano Heredia y la Unidad de Desarrollo de la Investigación, se solicitó un permiso para la ejecución del mismo a través del Departamento de Enfermería al cual pertenece el Centro de Crecimiento y Desarrollo - Lactancia materna.

Los criterios de inclusión fueron todas las madres y sus niños biológicos menores de 72 meses que acudieron al Centro de Crecimiento y DesarrolloLactancia Materna del HNCH, Lima- Perú en el año 2014 y los de exclusión niños de ambos sexos menores de 72 meses de edad que no presentaron piezas dentarias, padres o apoderados que no firmaron el Consentimiento Informado, madres o niños que presentaron alguna condición sistémica que comprometa su salud.

Antes de llevar a cabo la investigación se procedió a realizar un piloto, el cual tuvo como finalidad la calibración del examinador, se tuvo como Gold estándar a una especialista en odontopediatría y en estudios epidemiológicos de salud oral, obteniéndose una muy buena concordancia mediante la prueba de kappa mayor a 0,81 .

Una vez explicado el procedimiento a realizarse, su propósito y de recibir una respuesta afirmativa por parte de la madre del niño, que cumpla con los criterios de inclusión para realizar el estudio, se procedió a la firma del Consentimiento Informado por parte de la madre, el cual permitió la participación de las madres y los niños. Una vez firmado, se examinó a cada madre, todo fue registrado en la ficha epidemiológica con el índice de CPOD. El llenado de esta ficha se empezó especificando la presencia o ausencia de piezas dentarias, a continuación, se detalló la presencia de lesiones cariosas, obturaciones, etc. de acuerdo al índice de ceod/CPOD.

La ficha utilizada para el registro de datos contuvo datos generales del sujeto a evaluar como: edad, género, grado de instrucción y un registro de caries dental con el índice CPOD y ceod.

Se construyó una base de datos para su posterior análisis. El análisis univariado consistió en la frecuencia relativa y absoluta de las variables cualitativas, se obtuvieron promedios y desviaciones estándar de las variables cuantitativas. Para el análisis bivariado, se usó la prueba de Chi-cuadrado corregida por Yates y Fisher. Se determinó la distribución $\mathrm{z}$ de los datos cuantitativos mediante la prueba de Kolmogorov-Smirnov, por ello, se emplearon las pruebas no paramétricas de U de Mann Whitney, Kruskal Wallis y Correlación de Spearman. El presente estudio tuvo un nivel de confianza de $95 \%$ y un $\mathrm{p}<0,05$. Los datos fueron procesados utilizando el programa SPSS 20.0.

Para la realización del presente estudio, los sujetos de estudio firmaron un Consentimiento Informado, se conservó en anonimato a los participantes al otorgársele un código a cada uno, y se ejecutó luego de la aprobación del Comité Institucional de Ética de la Universidad Peruana Cayetano Heredia y del Comité Institucional de Ética del Hospital Nacional Cayetano Heredia.

\section{RESULTADOS}

El presente estudio se realizó en los meses de noviembre y diciembre del 2014 en el Centro de Crecimiento y Desarrollo-Lactancia Materna del $\mathrm{HNCH}$, Lima- Perú. El análisis se realizó sobre una muestra de 204 sujetos, siendo 102 madres y 102 niños. De los niños, $55.9 \%$ fueron de sexo masculino y el $44,1 \%$ femenino. De las madres, $74,5 \%$ habían terminado la secundaria completa y $23,5 \%$ educación superior. En cuanto a las edades, en las madres se tuvo una media de 29 años y en sus hijos de 16.7 meses (tabla 1) 
Tabla 1. Características sociodemográficas de las madres y sus hijos menores de 72 meses que acuden al Centro de Crecimiento y Desarrollo - Lactancia Materna del Hospital Nacional Cayetano Heredia, Lima- Perú.

\begin{tabular}{|c|c|c|c|}
\hline \multicolumn{2}{|c|}{ CARACTERÍSTICAS } & $\mathbf{n}$ & $\%$ \\
\hline \multicolumn{4}{|c|}{ Grado de instrucción de la madre } \\
\hline & Secundaria completa & 76 & 74.5 \\
\hline & Superior técnica & 2 & 2.0 \\
\hline & Superior universitaria & 24 & 23.5 \\
\hline \multicolumn{4}{|l|}{ Sexo del hijo } \\
\hline & Masculino & 57 & 55.9 \\
\hline & Femenino & 45 & 44.1 \\
\hline Edad de la madre (años) ${ }^{\circ}$ & & 29.0 & 7.6 \\
\hline Edad del hijo (meses) ${ }^{\circ}$ & & 16.7 & 11.3 \\
\hline
\end{tabular}

En cuanto a la prevalencia de caries dental, el $59.8 \%$ presentó lesiones de caries dental en las madres y $9.8 \%$ en los niños. En experiencia de caries dental, se encontró en las madres un CPOD promedio de 6,8 $(\mathrm{DE}=4,6)$ y un CPOS promedio de $16,7(\mathrm{DE}=15,8)$. En los niños se encontró que el índice ceod y ceos promedio de 0.3 en ambas $(\mathrm{DE}=1.1$ en el ceod $\mathrm{y}$ $\mathrm{DE}=1.2$ en ceos) (tabla 2).

Por otro lado, encontramos que el $39,2 \%$ de madres sin caries dental tenía también hijos sin caries dental y el $1 \%$ hijos con caries. En cuanto a las madres con caries dental, el 51\% tenía hijos sin caries dental y el $8.8 \%$ hijos con caries (tabla 3 ).

En cuanto a la experiencia de caries de la madre según su grado de instrucción se encontró un CPOD de $6.5,7.5,7.6$ y un CPOS de 16.7, 14.5, 16.9 para las madres con secundaria completa, educación superior técnica y universitaria, respectivamente. Según el sexo de su hijo, se encontró un CPOD de $6.3(\mathrm{DE}=4.4)$ para las madres con hijos y CPOD de $7.3(\mathrm{DE}=4.8)$ para las madres con hijas. Con el CPOS se encontró de 14.4 y 19.7 para las madres con hijos de sexo masculino y femenino, respectivamente. De igual forma, la experiencia de caries de los hijos se relacionó con el grado de instrucción de la madre y se encontró un ceod y un ceos de $0.4(\mathrm{DE}=1.2 \mathrm{y}$ $\mathrm{DE}=1.4$, respectivamente) de los hijos de madres con secundaria completa; y un ceod y ceos de 0.0 , de hijos de madres con estudios superior técnicos y universitarios. En cuanto al sexo, se encontró en niños de sexo masculino un ceod de $0.2(\mathrm{DE}=1.1)$ y ceos de $0.3(\mathrm{DE}=1.4)$; en las niñas un ceod y un ceos de $0.4(\mathrm{DE}=1.0)$ (tabla 4).

En cuanto a la prevalencia de caries de las madres, las madres sin caries dental tuvieron una edad promedio de 27.7 años $(\mathrm{DE}=7.1)$ y sus hijos de 14.1 meses $(\mathrm{DE}=7.5)$. Además, el $28.4 \%, 1 \%$ y el $10.8 \%$ tuvieron educación secundaria, superior técnica y universitaria, respectivamente. El 22.5\% de madres sin caries dental tenían hijos y el $17.6 \%$ hijas. Las madres con caries dental tenían una edad promedio de 29,8 años (DE=7.8) y sus hijos unos 18.4 meses $(\mathrm{DE}=13)$. Además, el $46,1 \%, 1 \% \mathrm{y}$ el $12,7 \%$ tenían educación secundaria, superior técnica y universitaria, respectivamente. El 33,3\% de madres, hijos de sexo masculino y el $26.5 \%$ de sexo femenino. Por otro lado, de los hijos sin caries dental, $52.9 \%$ fueron de sexo femenino y el $37,3 \%$ de sexo masculino, tuvieron una edad promedio de 14.4 meses $(\mathrm{DE}=7,7)$ y madres con una edad promedio de 28.3 años $(\mathrm{DE}=6,9)$. Y el 64,7\%, 2\% y $23,5 \%$ de sus madres tenían educación secundaria, superior técnica y universitaria, respectivamente. Los hijos con caries dental, fueron el 6,9\% de sexo femenino y el 2,9\% de sexo masculino, tuvieron una edad promedio de 37,6 meses $(\mathrm{DE}=16,7)$ y madres con un promedio de 35,2 años $(\mathrm{DE}=10,9)$. El 10\% de las madres con hijos con caries tenía educación secundaria (tabla 4). 
Tabla 2. Prevalencia y experiencia de caries dental de las madres y sus hijos menores de 72 meses que acuden al Centro de Crecimiento y Desarrollo Lactancia Materna del Hospital Nacional Cayetano Heredia, Lima- Perú

\begin{tabular}{|c|c|c|}
\hline CARIES DENTAL & $\mathbf{X}$ & DE \\
\hline \multicolumn{3}{|l|}{ Prevalencia de caries dental de las madres ${ }^{\circ}$} \\
\hline Sin caries dental & 41 & 40.2 \\
\hline Con caries dental & 61 & 59.8 \\
\hline \multicolumn{3}{|l|}{ Prevalencia de caries dental de los niños ${ }^{\circ}$} \\
\hline Sin caries dental & 92 & 90.2 \\
\hline Con caries dental & 10 & 9.8 \\
\hline \multicolumn{3}{|l|}{ Experiencia de caries dental de las madres } \\
\hline DC & 1.6 & 1.9 \\
\hline DP & 2.0 & 2.9 \\
\hline DO & 3.2 & 3.1 \\
\hline CPOD & 6.8 & 4.6 \\
\hline $\mathrm{SC}$ & 3.0 & 4.9 \\
\hline SP & 9.8 & 14.1 \\
\hline SO & 4.0 & 4.2 \\
\hline CPOS & 16.7 & 15.8 \\
\hline \multicolumn{3}{|l|}{ Experiencia de caries dental de los niños } \\
\hline $\mathrm{dc}$ & 0.3 & 1.1 \\
\hline de & 0.0 & 0.0 \\
\hline do & 0.0 & 0.0 \\
\hline ceod & 0.3 & 1.1 \\
\hline $\mathrm{sc}$ & 0.3 & 1.2 \\
\hline se & 0.0 & 0.0 \\
\hline so & 0.0 & 0.0 \\
\hline ceos & 0.3 & 1.2 \\
\hline
\end{tabular}

Tabla 3. Asociación de caries dental de las madres y sus hijos menores de 72 meses que acuden al Centro de Crecimiento y Desarrollo - Lactancia Materna del Hospital Nacional Cayetano Heredia, Lima- Perú

\begin{tabular}{|c|c|c|c|c|c|c|}
\hline \multirow[t]{3}{*}{ CARIES DENTAL } & \multicolumn{6}{|c|}{ Prevalencia de caries dental de sus niños } \\
\hline & \multirow{2}{*}{$\begin{array}{l}\text { Sin caries dental } \\
\mathbf{n}\end{array}$} & & \multicolumn{2}{|c|}{ Con caries dental } & \multirow{2}{*}{$\mathbf{p}$} & \\
\hline & & $\%$ & n & $\%$ & & \\
\hline \multicolumn{7}{|c|}{ Prevalencia de caries dental de las madres } \\
\hline & Sin caries dental & 40 & 39.2 & 1 & 1.0 & \multirow{2}{*}{$0.05^{*}$} \\
\hline & Con caries dental & 52 & 51.0 & 9 & 8.8 & \\
\hline
\end{tabular}

En cuanto a la prevalencia de caries dental por rangos de edades en los niños menores de 72 meses, encontramos que ningún niño $<12$ meses presentó caries dental. Sin embargo, presentaron caries dental en las edades de 13-24 meses $(5,1 \%)$, de $25-36$ meses $(30.8 \%)$, de $37-46$ meses $(50 \%)$, y la totalidad de niños de 47-56. En la experiencia de caries dental por rangos de edad en los niños menores de 72 meses, 


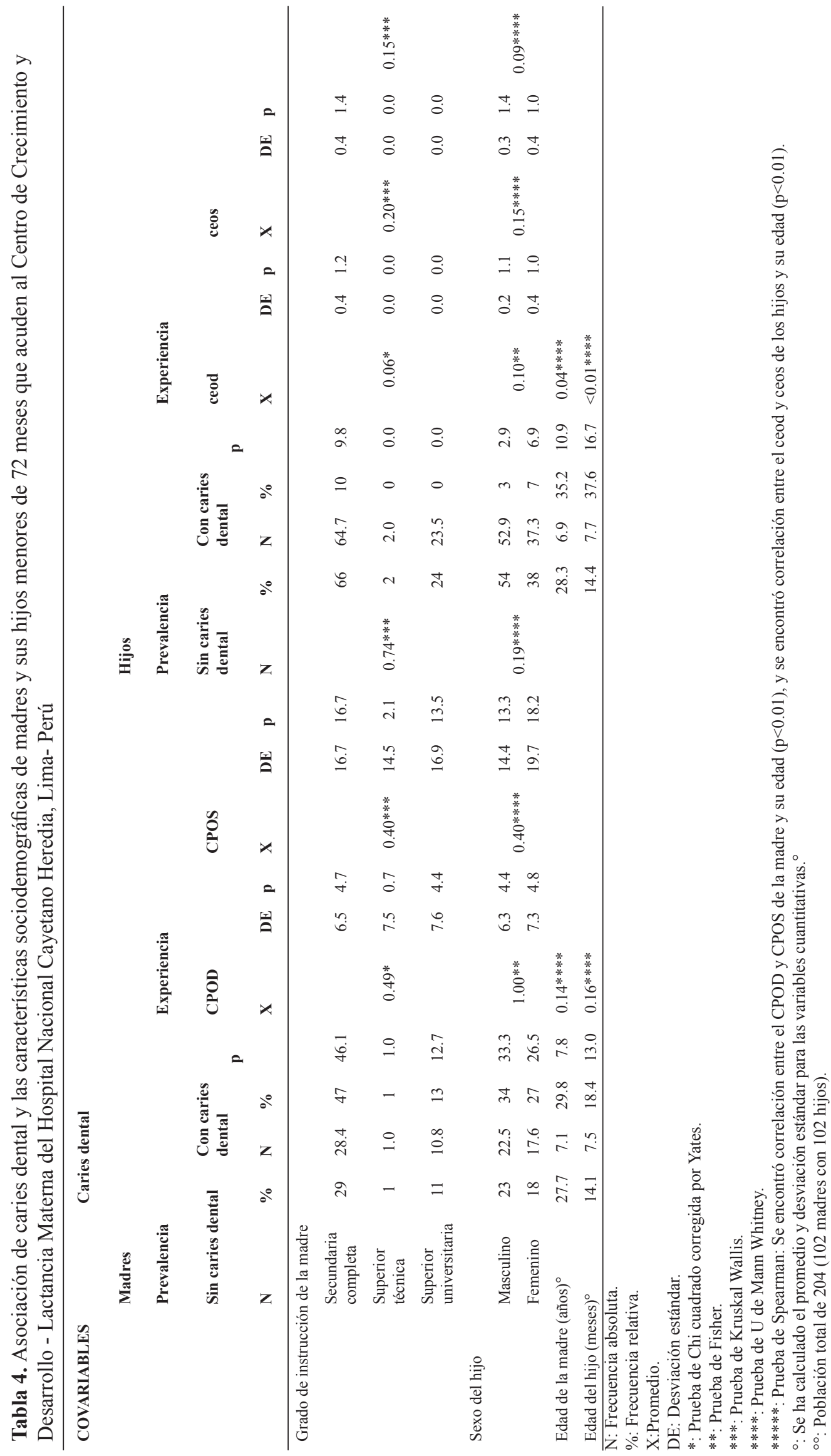


Tabla 5. Asociación de la prevalencia de caries dental y edad en meses de los hijos acuden al Centro de Crecimiento y Desarrollo - Lactancia Materna del Hospital Nacional Cayetano Heredia, LimaPerú en el año 2014.

\begin{tabular}{|c|c|c|c|c|c|}
\hline \multirow{3}{*}{ EDAD DEL HIJO (MESES) } & \multicolumn{5}{|c|}{ Prevalencia de caries dental de los hijos } \\
\hline & \multirow{2}{*}{$\begin{array}{c}\text { Sin caries dental } \\
\mathbf{n}\end{array}$} & \multicolumn{3}{|c|}{ Con caries dental } & \multirow{2}{*}{$\mathbf{p}^{*}$} \\
\hline & & $\%$ & $\mathbf{n}$ & $\%$ & \\
\hline De 0 a 12 meses & 45 & 100.0 & 0 & 0.0 & \\
\hline De 13 a 24 meses & 37 & 94.9 & 2 & 5.1 & \\
\hline De 25 a 36 meses & 9 & 69.2 & 4 & 30.8 & $<0.01$ \\
\hline De 37 a 48 meses & 1 & 50.0 & 1 & 50.0 & \\
\hline De 49 a 60 meses & 0 & 0.0 & 3 & 100.0 & \\
\hline
\end{tabular}

observamos en los niños $<12$ meses un ceod y ceos de 0 , de 13-24 meses un ceod de $0.3(\mathrm{DE}=1,3)$ y ceos de 0.3 ( $\mathrm{DE}=1.6)$, de $25-36$ meses un ceod de 0,7 $(\mathrm{DE}=1,4)$ y ceos de $0,8(\mathrm{DE}=1,4)$, de $37-48$ meses un ceod y ceos de $0,5(\mathrm{DE}=0,7)$, y, de 49-60 meses un ceod de $2.3(\mathrm{DE}=1,5)$ y ceos de $3(\mathrm{DE}=1)($ tabla 5$)$.

\section{DISCUSIÓN}

Las enfermedades que afectan a la cavidad oral como la caries dental, son enfermedades complejas que están originadas por la interacción de diferentes factores, y para su análisis se deben considerar la interacción de ellos tales como los componentes genéticos, epigenéticos, ambientales, culturales, sociales y locales $(5,6,7)$.

Las características sociodemográficas influencian la salud del niño, razón por la cual, en este estudio las asociamos con la prevalencia y la experiencia de caries. Encontramos que la mayoría de madres tenían secundaria completa, e hijos de sexo masculino. La edad promedio de las madres fue 29 años y la de los hijos 16,7 meses.

Al comparar estudios con los resultados obtenidos en la prevalencia de caries en madres que fue de un $59.8 \%$ y la de los hijos menores de 72 meses que fue de $9,8 \%$, encontramos que nuestros resultados mostraron valores menores, esto pudo explicarse al relacionar los resultados con el lugar de recojo de datos y al tomar en consideración que las madres asistentes al Centro de Crecimiento y DesarrolloLactancia Materna del $\mathrm{HNCH}$ podían ser personas preocupadas por su salud bucal y la de sus hijos, además, la mayoría de las madres tenían estudios secundarios completos y algunas estudios técnicos y universitarios concluidos, la literatura nos demuestra una fuerte relación entre determinantes sociales como nivel educacional y acceso a servicios de salud con el riesgo de caries (6). Estos factores explican también la baja prevalencia de caries dental encontrada en los niños menores de 72 meses.

En este estudio se midió la experiencia de caries dental a través del índice CPOD. Encontrando un CPOD promedio de 6.8 y un CPOS de 16.7 para las madres, en cuanto a los hijos, se encontró un ceod y un ceos promedio de 0,3 cada uno. Estudios realizados a nivel global evidencian diversos resultados en cuanto a la experiencia de caries dental en adultos, por ejemplo, en un estudio en adultos etíopes se encontró un CPOD promedio de 2,50 $\pm 2,21$ para adultos entre 15 a más de 20 años. (8) Lawal y col. realizaron un estudio con 430 personas en Nigeria encontrando un CPOD promedio de $0.7 \pm 1.6$ en adultos de entre 15 a más de 60 años (5). Drachev y col., realizaron un estudio con adultos jóvenes rusos de entre 18 y 25 años, encontrando un CPOD promedio de 7,58 (9) En Latinoamérica, Quinteros M. y col., encontraron un CPOD promedio de 15,29 en las 387 mujeres participantes con edades entre 25 y 34 años(10). Así mismo en Chile, en el 2012 se realizó un estudio nacional de prevalencia de caries y pérdida de dientes en adultos, en éste se evidenció un CPOD de 15.06 entre personas de 25- 44 años(11). El año 2019 en México, Arrieta-Vargas y col. encontraron un CPOD de 5,3 (12). En el Perú en el 2015, Espinoza 
encontró un CPOD promedio de 4,7 y un CPOS de 7,3(13). Medina encontró un promedio de CPOD de 14,05 (de 15,26 para las personas de sexo femenino y de 13,42 para los de sexo masculino) (14).

En cuanto a los hijos, Segovia A. y col encontraron un ceod de 1,5 +- 2,5 (15). Por otro lado, Davies G. y col encontraron un ceod promedio de 0,94 , encontrando promedios de ceod de 0,67 y 1,29 en el sureste y el noroeste, respectivamente (16). En Latinoamérica, Ueda E. y col encuentran un ceod promedio de 2,74 en 174 niños de entre 3 y 5 años (un ceod de 2,10 para niños de 3 años y de 3,51 para niños de 5 años) (17). Escobar G. y col encontraron un ceod promedio de 5,1 (ceod promedio de 0.67 al año y 5.9 a los 5 años) en un estudio realizado en niños de bajos recursos de 1 a 5 años (18). En el Perú, Villena R. et al encontraron un ceod promedio de 2,97 en una muestra de niños menores de 6 años de zonas urbano-marginales de Lima norte (19). Galvez C. encontraron un ceod promedio más alto (de 4,8) al evaluar la prevalencia de caries de 131 niños entre 3 y 5 años de edad de una institución educativa en Lima Metropolitana (20). Torres encontró en el 2010 un promedio similar la anterior, al evaluar 246 niños de entre 3 y 5 años en Huacho, el promedio de ceod fue 4,63 (1). Al igual que con la prevalencia, en la experiencia de caries de madres y de sus hijos menores de 72 meses encontramos resultados relativamente bajos al compararlos con estudios de poblaciones similares, esto se pudo explicar tomando en cuenta el sitio de recojo de datos y la preocupación de las madres por el cuidado de su salud oral y la salud bucal de sus hijos, además se consideró influyente el grado de instrucción de las madres y su acceso a los servicios de salud (6).

Sin embargo, en nuestro estudio no se encontró una asociación entre la salud bucal de las madres con la salud bucal de sus hijos menores de 72 meses. Esto podemos explicarlo, primero teniendo en cuenta que la mayoría de niños evaluados en este estudio tenían una edad muy corta y, por lo tanto, pocos dientes erupcionados al momento de la evaluación. En segundo lugar, debemos tomar en cuenta que la caries dental es una enfermedad multifactorial, es decir, que hay muchos factores relacionados con el desarrollo de la enfermedad; como la interrelación de las bacterias cariogénicas con el sustrato, la susceptibilidad del huésped, el tiempo y esto asociado a factores sociales, económicos y culturales, entre otros.
Gispert, Herrera y Felipe evaluaron a 159 niños de entre 6 meses y 1 año con el fin de analizar la influencia de la prevención indirecta en la incidencia bienal de caries en la primera infancia, y la relación entre la afectación por caries y el grado de infección por S. Mutans. Concluyendo que la incidencia de caries de los niños (al volver a ser evaluados 3 años después) depende del nivel de infección por $S$. Mutans de sus familiares, principalmente el de sus madres (21). Chaffe ycol concluyeron también que un elevado número de infección bacterial en madres, predice una infección bacteriana temprana y también una elevada incidencia de caries dental en los niños $(21,22)$. Kocatas et al., investigaron la prevalencia de caries de niños entre 15 y 35 meses y los factores maternos asociados, concluyendo que los factores relacionados con las madres como niveles de CPOD altos, fueron indicadores para identificar niños con alto riesgo de caries (23).

En países latinoamericanos Martinez y Rodriguez. realizaron un estudio con 60 binomios madre - hijo (los niños tenían $<7$ meses y ninguno tenía dientes erupcionados) a los que se les tomó muestras salivales, se analizó las cepas de S.Mutans de los binomios. Concluyeron que la transmisión vertical de los S.Mutans, confirmando la presencia de la bacteria en niños en los que aún no se ha producido la erupción dental (24). En el Perú, Guerrero realizó un estudio similar a los anteriores con 35 madres y 35 hijos en los que se encontró una asociación significativa entre cantidades altas de S.Mutans en las madres y las cantidades de S.Mutans en sus hijos (25).

Es por esto que, a pesar de que la madre tenga una influencia en la salud bucal del niño, al ser la persona más cercana a éste; al poder ser ésta la transmisora de las primeras bacterias cariogénicas, estos factores relacionados con la madre van a ser importantes más no determinantes en la aparición y desarrollo de la enfermedad.

Aaltonen seleccionó 327 infantes de 7 meses y los dividió en 2 grupos de acuerdo a la frecuencia de contactos salivares cercanos entre la madre y el infante, se tuvo un grupo $\mathrm{F}$ en el que se encontraron los niños con contactos salivares maternos frecuentes $\mathrm{y}$ un grupo $\mathrm{R}$ en el que se encontraron los niños con raros contactos salivares, de 5 a 7 años después, al examinar a los niños se encontró que los niños del grupo F tenían significativamente menos Streptococcus 
Mutans en saliva que los niños de grupo R. Además, la prevalencia de caries de los niños del grupo $\mathrm{F}$ fue de $19 \%$ y la del grupo R de $56 \%$ (26).

La educación, la edad y el género del individuo son factores que también evaluamos en este estudio. Diversos estudios han evidenciado su relación con la experiencia de caries dental. La educación es un factor importante en la salud bucal del individuo. Padres con mayor educación tendrían una mejor salud. Narang et al., encuentran que los padres con niveles más altos de educación tienen niños con menor experiencia de caries (27). En la misma India, Katageri et al., encuentran una mayor prevalencia de caries en niños con madres que poseen menores niveles de educación (28). Sin embargo, Ahmed et al., evidenciaron un aumento de la experiencia de caries en niños de 12 años en madres con educación superior, igual la prevalencia de caries (29). Por otro lado, en cuanto a la edad y el género, en Australia, Do y Roberts-Thomson evidenciaron un aumento en los promedios de CPOD a mayor edad (desde un 4.5 en el grupo de 15-34 a un 24.3 en el de 75 años a más) y un promedio de experiencia de caries mayor en las mujeres que en los hombres adultos del estudio, además encontraron que la prevalencia de caries al aumentar la edad no difería notablemente (30).

En el Perú, Medina encuentra una mayor experiencia de caries en mujeres que en hombres; en cuanto a la edad, evidencia un aumento del CPOD y CPOS en los grupos de mayor edad (14).

En el estudio se encuentran ciertas limitaciones que se vieron expresadas en diversos aspectos, como por ejemplo, la diferencia entre la experiencia y prevalencia de caries encontradas con los datos encontrados en la bibliografía, esto pudo explicarse tomando en cuenta que en el índice CPOD/ceod no se consideran las manchas blancas como una lesión de caries, debido a que otros estudios han demostrado que al considerarlas, se podría sobreestimar la enfermedad. Otra de las limitaciones del estudio recae en que el sitio de recojo de datos también pudo estar involucrado en la diferencia de los resultados en comparación con los de la literatura debido a que los asistentes al Centro de Crecimiento y Desarrollo - Lactancia Materna fueron en su mayoría niños menores de 1 año, disminuyendo esto su probabilidad de tener caries; además, el número de asistentes fue bastante reducido, lo que no nos permitió tener una muestra más grande y más variable. Además, el que el recojo de datos se haya dado en un hospital nacional como el $\mathrm{HNCH}$ pudo influenciar los resultados al suponer que las madres eran personas que suelen preocuparse por su salud general y por extensión, por su salud bucal.

La importancia del estudio radica en que los odontólogos deben estar al tanto de las enfermedades bucodentales que afectan a los pacientes. La caries dental, al ser la más prevalente, es sobre la que más informados debemos estar. Así como en los factores asociados a la misma, con el fin de poder educar a nuestros pacientes y buscar la prevención de estas enfermedades. Es así que, en este estudio, se evidenció la prevalencia de caries en menores de 5 años y sus respectivas madres, una relación importante debido a la gran influencia de la madre en la vida y salud de sus pequeños.

\section{CONCLUSIONES}

Al evaluar un grupo de madres y sus hijos menores de 72 meses de edad que acudieron al Centro de Crecimiento y Desarrollo y Crecimiento - Lactancia materna en el Hospital Nacional Cayetano Heredia, Lima - Perú; se encontró que no existió asociación entre caries dental del grupo de madres y de sus hijos menores de 72 meses evaluados. De manera específica pudimos concluir que la edad promedio de madres fue 29 años y la mayoría tuvo educación secundaria. La edad promedio de hijos fue 16.7 meses, siendo en la mayoría de sexo masculino. Además, la mayoría de madres presentaron lesiones de caries, sin embargo, la mayoría de hijos menores de 72 meses no presentó lesiones de caries dental. Finalmente, se encontró asociación entre la prevalencia de caries dental de los hijos y su edad, y entre la prevalencia de caries dental de las madres y sus edades.

\section{Correspondencia:}

Milagros Falcón

Jr.Morro Solar \#312 dpt.502 -Santiago de Surco

Lima, Perú

Correo electrónico: milafalcona@gmail.com 


\section{REFERENCIAS BIBLIOGRÁFICAS}

1. Pombo A. Relevancia clínica del cepillado bucodental infantil y su relación con los hábitos higiénicos y nutricionales del niñoy los conocimientos odontológicos de sus padres. Tesis doctoral. La Coruña:Departamento de Medicina, Universidade da Coruña; 2009.

2. Organización Mundial de la Salud. La Organización Mundial de la Salud publica un nuevo informe sobre el problema mundial de las enfermedades bucodentales. Ginebra: Organización Mundial de la Salud; 2004. (Citado el 10 de setiembre del 2020). Disponible en: http://www.who.int/mediacentre/news/releases/2004/ pr15/es/

3. Aguirre G, Fernandez R, Escobar W. Prevalencia de caries dental y necesidad de tratamiento según ICDAS y CPO en escolares de El Salvador. Horizonte sanitario. 2018;17(3):209-216.

4. Chumpitaz-Cerrate V, Bellido-Meza JA, ChávezRimache L, Rodríguez-Vargas C. Influencia del uso de inhaladores sobre la caries dental en pacientes pediátricos asmáticos: Estudio de casos y controles. Arch Argent Pediatr. 2020;118(1):38-43.

5. Lawal F, Alade O. Dental caries experience and treatment needs of an adult female population in Nigeria. Afri Health Sci. 2017;17(3): 905-911.

6. Fresno M, Geldes G, Estay J, Martin J. Prevalencia, severidad de caries dental y necesidad de tratamiento restaurador en escolares de 6 a 12 años de la provincia de Santiago, región Metropolitana. Rev. Clin. Periodoncia Implantol. Rehabil Oral Vol. 2019;12(2):81-86.

7. Diaz S, Ramos K, Arrieta K. Asociación del nivel de riesgo familiar total y caries dental en escolares de La Boquilla, Cartagena. Av enferm. 2013;31(2): 43-52

8. Sehdev B, Muruts L, Ganji KK. Prevalence of tooth decay and associated factors among Ethiopian patients. Pesqui Bras Odontopediatria Clín Integr. 2020; 20:e4835.

9. Drachev S, Brenn T, Trovik T. Dental caries experience and determinants in young adults of the Northern state Medical University, Arkhangelsk, North-West Russia: A cross-sectional study. BMC Oral Health. 2017; 17:136.

10. Quinteros M, Cáceres D, Soto A, Mariño R, Giacaman $\mathrm{R}$. Caries experience and use of dental services in rural and urban adults and older adults from central Chile. 2014. (Citado el 28 de junio del 2015) Disponible en: http://www.researchgate.net/publication/264744140

11. Urzua I, C Mendoza C, Arteaga O, et al.Dental caries prevalence and tooth loss in Chilean adult population: First national dental examination survey. Int J Dent. 2012; 2012: 810170. doi: 10.1155/2012/810170

12. Arrieta-Vargas L. et al. Prevalencia de caries y factores asociados: estudia transversal en estudiantes de preparatoria de Chipalcingo, Guerrero, México. Revista Odontológica Mexicana. 2019;23 (1): 31-41.

13. Espinoza M, Leon-Manco RL. Prevalencia y experiencia de caries dental en estudiantes según facultades de una universidad particular peruana. Rev Estomatol Herediana. 2015.25(3):187-193.

14. Medina JF, Alvarado S. Prevalencia de caries dental y necesidad de tratamiento en pacientes adultos con demanda de atención diagnóstica. Odontol Clín Cient. 2012;11(2):155-158.

15. Segovia A, Estrella R, Medina CE, Maupomé G. Severidad de caries y factores asociados en prescolares de 3 - 6 años de edad en Campeche, México. Rev Salud Pública. 2004;7(1):56-69.

16. Davies G. National Dental Epidemiology Programme for England: oral health survey of five-year-old children 2012 A report on the prevalence and severity of dental decay. London: Public Health England; 2013. (Consultado el 02 de Julio del 2015) Disponible en: $\quad$ https://communitydentalservices.co.uk/wpcontent/uploads/2019/07/Dental-Surveys-OralHealth-5yr-old-children-2012-final-report-gatewayapproved.pdf

17. Ueda E, Dezan C, Frozzard W, Salomao F, Morita M. Prevalence of dental caries in 3 and 5 year old children living in a small Brazilian City. J Appl Oral Sci. 2004;12(1):34-8.

18. Escobar G, Ramirez B, Franco A, Tamayo A. Castro JF. Experiencia de caries dental en niños de $1-5$ años de bajos ingresos. Medellín. Colombia. Rev CES Odont. 2009;22(1):21-8

19. Villena R, Pachas F, SánchezY, Carrasco M. Prevalencia de caries de infancia temprana en niños menores de 6 años de edad, residentes en poblados urbano-marginales de Lima Norte. Rev Estomatol Herediana. 2011;21(2):79-86

20. Galvez C. Prevalencia de caries dental en niños con dentición decidua usando índices CEO y el sistema internacional de detección y valoración de caries (ICDAS II). Tesis pregrado. Lima, Perú. Universidad Norbert Wiener; 2013.

21. Gispert E, Herrera M, Felipe I. Prevención indirecta de caries en la infancia temprana. Rev Cubana Estomatol. 2006; 43(4):0-0.

22. Chaffe BW, Gansky SA, Weintraub SK, Featherstone JDB, Ramos-Gomez FJ. Maternal oral bacterial levels predict early childhood caries development. J Dent Res. 2014;93(3):238-244.

23. Kocatas N, Eronat N, Cogulu D, Uzel A, Aksit S. Association of maternal - child characteristics as a factor in early childhood caries and salivary bacterial counts. J Dent Child. 2006; 73(2):105-111.

24. Martínez MC, Rodríguez A. Estudio de las cepas de estreptococos del grupo mutans presentes en binomios madre- hijo. Rev Fac Odontol Univ Antioq. 2009; 


\section{1(2): 177-85.}

25. Guerrero M. Contaminación directa madre-niño a través de la saliva. Tesis de pregrado. Lima, Perú: Universidad Nacional Federico Villarreal; 2003.

26. Aaltonen A. Association between mother infant salivary contacts and caries resistance in children: A cohort study. Pediatric Dentistry. 1994;16(2):110-16.

27. Narang R, Mittal L, Jha K, Anamika, Roseka. Caries experience and its relationship with parents education, occupation and socio economic status of the family among 3-6 years old preschool children of Sri Ganganagar City, India. Open Journal of Dentistry and Oral Medicine. 2013;1(1):1-4.
28. Katageri A, Arora R, Bhayya D, Patil K. Prevalence of dental caries and its association to maternal education as a risk indicator: A cross section study in Udaipur - Rajasthan. Scholars Journal of Applied Medical Sciences. 2014;2(3):983-6.

29. Ahmed N, Astrom A, Skaug N, Petersen E. Dental caries prevalence and risk factors among 12 years old schoolchildren from Baghdad, Iraq: a post-war survey. International Dental Journal. 2007;57:36-44.

30. Do LG, Roberts-Thomson KF. Dental caries experience in the Australian adult population. Australian Dental Journal. 2007;52(3):249-51.

Recibido : 15-08-2020

Aceptado : 10-12-2020 